\title{
Darboux Transformation and Exact Solutions for High Order Nonlocal Coupled AKNS System
}

\author{
Xiangpeng Xin*, Zengxin Guo, Yanxin Hu, Linlin Zhang \\ School of Mathematical Sciences, Liaocheng University, Liaocheng, China \\ Email: *xinxiangpeng@lcu.edu.cn
}

How to cite this paper: Xin, X.P., Guo, Z.X., Hu, Y.X. and Zhang, L.L. (2021) Darboux Transformation and Exact Solutions for High Order Nonlocal Coupled AKNS System. Journal of Applied Mathematics and Physics, 9, 2592-2608. https://doi.org/10.4236/jamp.2021.911167

Received: September 15, 2021

Accepted: October 31, 2021

Published: November 3, 2021

Copyright $\odot 2021$ by author(s) and Scientific Research Publishing Inc. This work is licensed under the Creative Commons Attribution International License (CC BY 4.0).

http://creativecommons.org/licenses/by/4.0/

\begin{abstract}
In this paper, we investigate an integrable high order nonlocal coupled Ablowitz-Kaup-Newell-Segur (AKNS) system for the first time. With the aid of Lax pair of this nonlocal system, Darboux transformation (DT) and new soliton-like solutions are obtained. Different from local equations, Darboux transformation of nonlocal systems needs to meet certain conditions. In this article, under the condition of symmetry reduction, the components of Darboux transformation need to satisfy $b_{21}^{[k]}(x, t)=-b_{12}^{[k]}(-x,-t), b_{23}^{[k]}(x, t)=-b_{14}^{[k]}(-x,-t)$. In order to study the dynamic information of the solutions, the images of the solutions are given.
\end{abstract}

\section{Keywords}

High Order Nonlocal Equation, Darboux Transformation, Exact Solution

\section{Introduction}

In the past decades, a number of researches involved in applied mathematics and theoretical physics are concerned with integrable systems. The KdV, the nonlinear Schrödinger, the $\mathrm{mKdV}$, the sine-Gordon equations, etc. are well-known integrable equations, and they can all be derived from the AKNS system. Many scholars have studied these equations from different fields and got many excellent results. An important research topic is to find the exact solutions of these systems, especially their soliton solutions or soliton-like solutions. So far, many effective methods have been developed, such as, inverse scattering method [1] [2] [3], Classical and nonclassical lie group method [1] [2] [3], Darboux transformation method [4] [5] [6] [7] [8], bilinear method [9] [10] [11] [12], etc.

It is well known that symmetry is one of the important characteristics of integrable systems. By using Lie group method, the system can be reduced and 
solved, the conservation law of the system can also be constructed at the same time. Lie symmetry is also called continuous symmetry because its variables are transformed in the following form, $x^{*}=x+\varepsilon \xi, t^{*}=t+\varepsilon \tau, u^{*}=u+\varepsilon \psi$, where $\varepsilon$ is infinitesimal. However, many facts show that two or more events occur at different places and times but are closely related to each other. For example, the reduction of Arctic sea ice in the summer of 2007 is considered to be the reason for the heavy winter freezing rain in southern China in 2008. To explain these events, nonlocal models are proposed, such as nonlocal nonlinear Schrödinger equation [13],

$$
i q_{t}(x, t)+q_{x x}(x, t)+2 q^{2}(x, t) q^{*}(-x, t)=0,
$$

was proposed by Ablowitz, where * represents complex conjugation. One can see from Equation (1.1) that $q$ is not only a function of $x$ but also a function of $-X$, that is to say, the solution $q(x, t)$ evaluated at $x$ requires information from $-x$ via the term $q^{*}(-x, t)$. For system (1.1), the exact solution and the conservation law are studied by the inverse scattering method. Before long, the nonlocal modified KdV systems, the discrete nonlocal NLS system, nonlocal sine-Gordon equation and the nonlocal Davey-Stewartson systems were also proposed. In general, nonlocal equations fall into three categories: 1) full $P T$ symmetry; 2) partial $P T$ symmetry; 3) partial reverse space-time symmetry. For this new type of system, many scholars have studied the integrability, exact solution [14] [15], conservation law and other aspects of the system, and got a lot of excellent results. Because nonlocal systems play an important role in mathematics and physics, more and more experts are attracted to study this kind of equations [15] [16] [17] [18] [19].

Constructing the exact solutions of these nonlinear nonlocal equations can help to verify the numerical solutions and help to analyze the stability of the solutions. However, it is very difficult to solve them because of their high nonlinearity. Over the past few decades, significant progress has been made in many methods. The Darboux transform based on Lax pair has been proved to be one of the most effective algorithms for solving explicit solutions of nonlinear evolution equations. By using Darboux transformation method, soliton solutions, rogue-wave solutions of those nonlocal integrable equations are given [20]-[25].

This paper is arranged as follows: In Section 2, the high order nonlocal coupled AKNS system is constructed by using the linear spectral problem. In Section 3, the process of Darboux transformations is introduced in detail. Exact 1 -fold and $\mathrm{N}$-fold solutions are obtained. In Section 4, using the trivial seed solutions, the exact solutions of the high order nonlocal coupled AKNS system are constructed. In order to study the properties of the solution, the appropriate parameters are selected and the corresponding images are made. Finally, some conclusions and discussions are given in Section 5.

\section{High Order Nonlocal Coupled AKNS System}

In this section, we will construct the high order nonlocal coupled AKNS system. 
In fact, the starting point is the AKNS scattering problem [26],

$$
\begin{aligned}
& \phi_{x}=U \phi, \\
& \phi_{t}=V \phi,
\end{aligned}
$$

$\phi=\left(\phi_{1}(x, t), \phi_{2}(x, t), \phi_{3}(x, t), \phi_{4}(x, t)\right)^{\mathrm{T}}$, and the matrices $U, V$ have the following form matrixes,

$$
U=\left(\begin{array}{cccc}
-\lambda & P & -\lambda & R \\
Q & \lambda & S & \lambda \\
0 & 0 & -\lambda & P \\
0 & 0 & Q & \lambda
\end{array}\right), V=\left(\begin{array}{cccc}
A & B & E & F \\
C & -A & G & -E \\
0 & 0 & A & B \\
0 & 0 & C & -A
\end{array}\right),
$$

which was first introduced by Ma et al., A, B, C,E,F, $G$ have the following form,

$$
\begin{aligned}
A= & \alpha\left(\lambda^{3}-\frac{1}{4} p q_{x}+\frac{1}{4} q p_{x}-\frac{1}{2} \lambda p q\right), \\
B= & \alpha\left(-\lambda^{2} p+\frac{1}{2} \lambda p_{x}-\frac{1}{4} p_{x x}+\frac{1}{2} p^{2} q\right), \\
C= & \alpha\left(-\lambda^{2} q-\frac{1}{2} \lambda q_{x}-\frac{1}{4} q_{x x}+\frac{1}{2} p q^{2}\right), \\
E= & \alpha\left(\lambda\left(p q-\frac{p s}{2}-\frac{q r}{2}\right)+\frac{3}{4} p q_{x}-\frac{3}{4} q p_{x}-\frac{1}{4} p s_{x}+\frac{1}{4} q r_{x}-\frac{1}{4} r q_{x}+\frac{1}{4} s p_{x}\right) \\
& +\beta\left(\lambda^{3}-\frac{1}{4} p q_{x}+\frac{1}{4} q p_{x}-\frac{1}{2} \lambda p q\right), \\
F= & \alpha\left(\lambda^{2}(p-r)-\lambda\left(p_{x}-\frac{1}{2} r_{x}+\frac{3}{4} p_{x x}-\frac{1}{4} r_{x x}-\frac{3}{2} p^{2} q+\frac{1}{2} p^{2} s+p q r\right)\right) \\
& +\beta\left(-\lambda^{2} p+\frac{1}{2} \lambda p_{x}-\frac{1}{4} p_{x x}+\frac{1}{2} p^{2} q\right), \\
G= & \alpha\left(\lambda^{2}(q-s)-\lambda\left(q_{x}-\frac{1}{2} s_{x}+\frac{3}{4} q_{x x}-\frac{1}{4} s_{x x}-\frac{3}{2} p q^{2}+\frac{1}{2} q^{2} r+p q s\right)\right) \\
& +\beta\left(-\lambda^{2} q+\frac{1}{2} \lambda q_{x}-\frac{1}{4} q_{x x}+\frac{1}{2} p q^{2}\right),
\end{aligned}
$$

where $p, q, r, s$ are four potentials, $\alpha$ is arbitrary constant and $\lambda$ is the spectral parameter.

The compatibility condition of the system (2.1) yields the zero curvature representation $U_{t}-V_{x}+[U, V]=0$, using this formula, one can obtain the following evolution equations,

$$
\begin{aligned}
p_{t}= & \frac{3}{2} \alpha p q p_{x}-\frac{1}{4} \alpha p_{x x x}, \\
q_{t}= & \frac{3}{2} \alpha p q q_{x}-\frac{1}{4} \alpha q_{x x x}, \\
r_{t}= & \frac{3}{4} \alpha p_{x x x}-\frac{1}{4} \alpha r_{x x x}-\frac{1}{4} \beta p_{x x x}+\frac{3}{2} \beta p q p_{x}-\frac{9}{2} \alpha p q p_{x} \\
& +\frac{3}{2} \alpha p q r_{x}+\frac{3}{2} \alpha p s p_{x}+\frac{3}{2} \alpha q r p_{x},
\end{aligned}
$$




$$
\begin{aligned}
s_{t}= & \frac{3}{4} \alpha q_{x x x}-\frac{1}{4} \alpha s_{x x x}-\frac{1}{4} \beta q_{x x x}+\frac{3}{2} \beta p q q_{x}-\frac{9}{2} \alpha p q q_{x} \\
& +\frac{3}{2} \alpha p q s_{x}+\frac{3}{2} \alpha q r q_{x}+\frac{3}{2} \alpha p s q_{x},
\end{aligned}
$$

system (2.4) is coupled mixed nonlinear Schrödinger equation, it possesses infinitely many symmetries, which constitute some infinite-dimensional Lie algebra.

In this section, we set a symmetry reduction to (2.4),

$$
\begin{aligned}
& q(x, t)=p(-x,-t), \\
& s(x, t)=r(-x,-t),
\end{aligned}
$$

the high order nonlocal coupled AKNS systemn can be obtained,

$$
\begin{aligned}
p_{t}= & \frac{3}{2} \alpha p p_{x} p(-x,-t)-\frac{1}{4} \alpha p_{x x x}, \\
r_{t}= & \frac{1}{4}\left(3 \alpha p_{x x x}-\alpha r_{x x x}-\beta p_{x x x}\right)+\frac{3}{2} p p_{x}(\beta p(-x,-t)-3 \alpha p(-x,-t) \\
& +\alpha r(-x,-t))+\frac{3}{2} \alpha p(-x,-t)(p r)_{x},
\end{aligned}
$$

The integrable high order nonlocal coupled AKNS system is constructed by using the symmetry reduction method. The following work is to construct the exact solution of the system (2.6). It is well known that the Darboux transform is an important method for solving the exact solution of integrable nonlinear systems. It is proved that this method is not only suitable for solving local equations, but also for solving nonlocal equations. In the next section, we will construct the Darboux transform and exact solution of the nonlocal integrable system (2.6).

\section{Darboux Transformation for High Order Nonlocal Coupled AKNS System}

In this section, we will construct the Darboux transformation (DT) by means of the lax pair of high order nonlocal coupled AKNS system. The idea of the DT is to construct a transformation such that the form of the lax pair remains unchanged. Similarly to classical DT, we take the following gauge transformation,

$$
\phi^{[1]}=T^{[1]} \phi .
$$

Assume that the new lax pair has the following form under the gauge transformation (4.1),

$$
\begin{aligned}
& \phi_{x}^{[1]}=U^{[1]} \phi^{[1]}, \\
& \phi_{t}^{[1]}=V^{[1]} \phi^{[1]},
\end{aligned}
$$

where

$$
\begin{aligned}
U^{[1]} & =\left(T_{x}^{[1]}+T^{[1]} U\right)\left(T^{[1]}\right)^{-1}, \\
V^{[1]} & =\left(T_{t}^{[1]}+T^{[1]} V\right)\left(T^{[1]}\right)^{-1},
\end{aligned}
$$

the next work is to find a matrix $T^{[1]}$ such that $U^{[1]}, V^{[1]}$ have the same forms as $U, V$, and $p, q, r, s$ are mapped into new potentials $p^{[1]}, q^{[1]}, r^{[1]}, s^{[1]}$, i.e. 


$$
U^{[1]}=\left(\begin{array}{cccc}
-\lambda & p^{[1]} & -\lambda & r^{[1]} \\
q^{[1]} & \lambda & s^{[1]} & \lambda \\
0 & 0 & -\lambda & p^{[1]} \\
0 & 0 & q^{[1]} & \lambda
\end{array}\right), \quad V^{[1]}=\left(\begin{array}{cccc}
A^{[1]} & B^{[1]} & E^{[1]} & F^{[1]} \\
& -A^{[1]} & G^{[1]} & -E^{[1]} \\
0 & 0 & A^{[1]} & B^{[1]} \\
0 & 0 & C^{[1]} & -A^{[1]}
\end{array}\right),
$$

where $A^{[1]}, B^{[1]}, C^{[1]}, E^{[1]}, F^{[1]}, G^{[1]}$ have the same forms as A, B, C, E, F, G (2.3), just like the forms of $U^{[1]}$.

In order to construct the form of the gauge matrix $T$, we suppose,

$$
T^{[1]}=\left(\begin{array}{cc}
T_{0} & T_{1} \\
0 & T_{0}
\end{array}\right),
$$

where $T_{0}$ and $T_{1}$ have the following forms,

$$
\begin{aligned}
& T_{0}=\left(\begin{array}{cc}
\lambda+b_{11}^{[1]}(x, t) & b_{12}^{[1]}(x, t) \\
b_{21}^{[1]}(x, t) & \lambda+b_{22}^{[1]}(x, t)
\end{array}\right), \\
& T_{1}=\left(\begin{array}{cc}
\lambda+b_{13}^{[1]}(x, t) & b_{14}^{[1]}(x, t) \\
b_{23}^{[1]}(x, t) & \lambda+b_{24}^{[1]}(x, t)
\end{array}\right),
\end{aligned}
$$

$b_{i j}^{[1]}, i=1,2 ; j=1,2,3,4$ are undetermined functions of $x$ and $t$.

By substituting (3.5) into (3.3), we obtain the transformations between $p, q, r, s$ and $p^{[1]}, q^{[1]}, r^{[1]}, s^{[1]}$ by balancing the order of $\lambda$,

$$
\begin{aligned}
& p^{[1]}(x, t)=p(x, t)+2 b_{12}^{[1]}(x, t), \\
& q^{[1]}(x, t)=q(x, t)-2 b_{21}^{[1]}(x, t), \\
& r^{[1]}(x, t)=r(x, t)+2 b_{14}^{[1]}(x, t), \\
& s^{[1]}(x, t)=s(x, t)-2 b_{23}^{[1]}(x, t),
\end{aligned}
$$

and

$$
\begin{aligned}
& \frac{\partial}{\partial x} b_{11}=p^{[1]} b_{21}-b_{12} q, \quad \frac{\partial}{\partial x} b_{12}=p^{[1]} b_{22}-b_{11} p, \\
& \frac{\partial}{\partial x} b_{13}=p^{[1]} b_{23}+r_{1} b_{21}-b_{12} s-b_{14} q, \quad \frac{\partial}{\partial x} b_{14}=p^{[1]} b_{24}+r_{1} b_{22}-b_{11} r-b_{13} p, \\
& \frac{\partial}{\partial x} b_{21}=q^{[1]} b_{11}-b_{22} q, \quad \frac{\partial}{\partial x} b_{22}=q^{[1]} b_{12}-b_{21} p, \\
& \frac{\partial}{\partial x} b_{23}=q^{[1]} b_{13}+s_{1} b_{11}-b_{22} s-b_{24} q, \quad \frac{\partial}{\partial x} b_{24}=q^{[1]} b_{14}+s_{1} b_{12}-b_{21} r-b_{23} p,
\end{aligned}
$$

because $q^{[1]}(x, t), p^{[1]}(x, t), r^{[1]}(x, t), s^{[1]}(x, t)$ are still solutions to the Equation (2.4), so we assume that these four new potentials satisfy the symmetry reduction condition $q^{[1]}(x, t)=p^{[1]}(-x,-t), s^{[1]}(x, t)=r^{[1]}(-x,-t)$. Using this condition, one can get,

$$
\begin{gathered}
b_{21}^{[1]}(x, t)=-b_{12}^{[1]}(-x,-t), \\
b_{23}^{[1]}(x, t)=-b_{14}^{[1]}(-x,-t),
\end{gathered}
$$

so the $N$-fold Darboux transformation for nonlocal couplings of AKNS equations (2.6) can be constructed, 


$$
\Phi^{[n]}=T_{n}(\lambda) \Phi=T^{[n]}(\lambda) T^{[n-1]}(\lambda) \cdots T^{[k]}(\lambda) \cdots T^{[1]}(\lambda) \Phi,
$$

the gauge transformation $T^{[k]}(\lambda)$ has a similar form to (2.4). Different from the Darboux transformation of local equations, the Darboux transformation of nonlocal equations needs to meet the following constraints,

$$
\begin{aligned}
& b_{21}^{[k]}(x, t)=-b_{12}^{[k]}(-x,-t), \\
& b_{23}^{[k]}(x, t)=-b_{14}^{[k]}(-x,-t), k=1,2, \cdots, N
\end{aligned}
$$

The relationship between new and old potentials is as follows,

$$
\begin{aligned}
& p^{[k]}=p+2 b_{12}^{[k]}(-x,-t), \\
& r^{[k]}=r+2 b_{14}^{[k]}(-x,-t) .
\end{aligned}
$$

Proposition 1. Under the action of transformation $T^{[1]}$ (3.5), the matrix $U^{[1]}$ determined by Equation (3.2) has the same form as $U$, that is,

$$
U^{[1]}=\left(\begin{array}{cccc}
-\lambda & p^{[1]} & -\lambda & r^{[1]} \\
q^{[1]} & \lambda & s^{[1]} & \lambda \\
0 & 0 & -\lambda & p^{[1]} \\
0 & 0 & q^{[1]} & \lambda
\end{array}\right),
$$

the transformations between $p, q, r, s$ and $p^{[1]}, q^{[1]}, r^{[1]}, s^{[1]}$ are given by Equation (3.6).

Proof: For convenience, we will write $T^{[1]}$ as $T$, according to formulas $T^{-1}=(\operatorname{det} T)^{-1} T^{*}$, and

$$
\left(T_{x}+T U\right) T^{*}=\left(\begin{array}{cccc}
f_{11}(\lambda) & f_{12}(\lambda) & f_{13}(\lambda) & f_{14}(\lambda) \\
f_{21}(\lambda) & f_{22}(\lambda) & f_{23}(\lambda) & f_{24}(\lambda) \\
0 & 0 & f_{33}(\lambda) & f_{34}(\lambda) \\
0 & 0 & f_{43}(\lambda) & f_{44}(\lambda)
\end{array}\right)
$$

It can be known by calculation that $f_{i j}(\lambda)$ are four or five times polynomials about $\lambda$, and it can be proved that $\lambda_{j}, j=1,2$ are the roots of $f_{i j}(\lambda)$.

Because $\lambda_{j}(j=1,2)$ are the roots of $\operatorname{det} T$, we can rewrite (3.12) as

$$
\left(T_{x}+T U\right) T^{*}=(\operatorname{det} T) P(\lambda),
$$

with

$$
P(\lambda)=\left(\begin{array}{cccc}
\sum_{i=0}^{5} p_{11}^{(i)} \lambda^{i} & \sum_{i=0}^{4} p_{12}^{(i)} \lambda^{i} & \sum_{i=0}^{5} p_{13}^{(i)} \lambda^{i} & \sum_{i=0}^{4} p_{14}^{(i)} \lambda^{i} \\
\sum_{i=0}^{4} p_{21}^{(i)} \lambda^{i} & \sum_{i=0}^{5} p_{22}^{(i)} \lambda^{i} & \sum_{i=0}^{4} p_{23}^{(i)} \lambda^{i} & \sum_{i=0}^{5} p_{24}^{(i)} \lambda^{i} \\
0 & 0 & \sum_{i=0}^{5} p_{33}^{(i)} \lambda^{i} & \sum_{i=0}^{4} p_{34}^{(i)} \lambda^{i} \\
0 & 0 & \sum_{i=0}^{4} p_{43}^{(i)} \lambda^{i} & \sum_{i=0}^{5} p_{44}^{(i)} \lambda^{i}
\end{array}\right),
$$

where undetermined $P_{i j}^{(k)}$ are not functions of $\lambda$. Because $T^{-1}=T^{*} \cdot(\operatorname{det} T)^{-1}$, so Equation (3.13) can be rewritten as, 


$$
T_{x}+T U=P(\lambda) T .
$$

Substitute Equation (3.5) and Equation (3.14) into Equation (3.15), by comparing the coefficients of the same power of $\lambda$ on both sides of Equation (3.15), the following system of equations can be obtained,

$$
\begin{gathered}
\lambda^{6}: p_{11}^{5}=0, p_{13}^{5}=0, p_{22}^{5}=0, p_{24}^{5}=0, p_{33}^{5}=0, p_{44}^{5}=0, \\
\lambda^{5}: p_{11}^{4}=0, p_{12}^{4}=0, p_{13}^{4}=0, p_{21}^{4}=0, p_{22}^{4}=0, p_{34}^{4}=0, p_{43}^{4}=0, \\
p_{13}^{4}=0, p_{44}^{4}=0, p_{14}^{4}=0, p_{23}^{4}=0, p_{24}^{4}=0, \\
\lambda^{4}: p_{11}^{3}=0, p_{12}^{3}=0, p_{21}^{3}=0, p_{22}^{3}=0, p_{33}^{3}=0, p_{34}^{3}=0, p_{44}^{3}=0, \\
p_{43}^{3}=0, p_{13}^{3}=0, p_{14}^{3}=0, p_{23}^{3}=0, p_{24}^{3}=0, \\
\lambda^{3}: p_{11}^{2}=0, p_{12}^{2}=0, p_{21}^{2}=0, p_{22}^{2}=0, p_{33}^{2}=0, p_{34}^{2}=0, p_{43}^{2}=0, \\
p_{44}^{2}=0, p_{13}^{2}=0, p_{14}^{2}=0, p_{23}^{2}=0, p_{24}^{2}=0, \\
\lambda^{2}: p_{12}^{1}=0, p_{21}^{1}=0, p_{34}^{1}=0, p_{43}^{1}=0, p_{14}^{1}=0, p_{23}^{1}=0, p_{11}^{1}=-1, \\
p_{33}^{1}=-1, p_{13}^{1}=-1, p_{22}^{1}=1, p_{44}^{1}=1, p_{24}^{1}=1, \\
\lambda^{1}: p_{12}^{0}=p+2 b_{12}=p^{[1]}, p_{34}^{0}=p+2 b_{12}=p^{[1]}, p_{21}^{0}=q-2 b_{21}=q^{[1]}, \\
p_{43}^{0}=q-2 b_{21}=q^{[1]}, p_{14}^{0}=r+2 b_{14}=r^{[1]}, p_{23}^{0}=s-2 b_{23}=s^{[1]}, \\
p_{22}^{0}=0, p_{11}^{0}=0, p_{33}^{0}=0, p_{44}^{0}=0, p_{13}^{0}=0, p_{24}^{0}=0,
\end{gathered}
$$

and the coefficients of $\lambda^{0}$ satisfies Equation (3.7). By substituting the above results into Equation (3.14), we can get,

$$
P(\lambda)=\left(\begin{array}{cccc}
-\lambda & p^{[1]} & -\lambda & r^{[1]} \\
q^{[1]} & \lambda & s^{[1]} & \lambda \\
0 & 0 & -\lambda & p^{[1]} \\
0 & 0 & q^{[1]} & \lambda
\end{array}\right),
$$

then from Equation (3.3), we can get $U^{[1]}=P(\lambda)$.

Using the same process, we can prove that $V^{[1]}$ has the same form as $V$ under the transformation.

Proposition 2. Under the action of transformation $T^{[1]}$ (3.5), the matrix $V^{[1]}$ determined by Equation (3.2) has the same form as $V$, that is,

$$
V^{[1]}=\left(\begin{array}{cccc}
A^{[1]} & B^{[1]} & E^{[1]} & F^{[1]} \\
C^{[1]} & -A^{[1]} & G^{[1]} & -E^{[1]} \\
0 & 0 & A^{[1]} & B^{[1]} \\
0 & 0 & C^{[1]} & -A^{[1]}
\end{array}\right)
$$

where $A^{[1]}, B^{[1]}, C^{[1]}, E^{[1]}, F^{[1]}, G^{[1]}$ have the form (3.4), and the transformations between $p, q, r, s$ and $p^{[1]}, q^{[1]}, r^{[1]}, s^{[1]}$ are given by Equation (3.6).

Proof: According to formulas $T^{-1}=(\operatorname{det} T)^{-1} T^{*}$, and

$$
\left(T_{t}+T V\right) T^{*}=\left(\begin{array}{cccc}
g_{11}(\lambda) & g_{12}(\lambda) & g_{13}(\lambda) & g_{14}(\lambda) \\
g_{21}(\lambda) & g_{22}(\lambda) & g_{23}(\lambda) & g_{24}(\lambda) \\
0 & 0 & g_{33}(\lambda) & g_{34}(\lambda) \\
0 & 0 & g_{43}(\lambda) & g_{44}(\lambda)
\end{array}\right)
$$


where $g_{i j}(\lambda)$ are six or seven times polynomials about $\lambda$, and $\lambda_{j}, j=1,2$ are the roots of $g_{i j}(\lambda)$. So we can rewrite (3.16) as

$$
\left(T_{t}+T U\right) T^{*}=(\operatorname{det} T) K(\lambda),
$$

with

$$
K(\lambda)=\left(\begin{array}{cccc}
\sum_{i=0}^{7} k_{11}^{(i)} \lambda^{i} & \sum_{i=0}^{6} k_{12}^{(i)} \lambda^{i} & \sum_{i=0}^{7} k_{13}^{(i)} \lambda^{i} & \sum_{i=0}^{6} k_{14}^{(i)} \lambda^{i} \\
\sum_{i=0}^{6} k_{21}^{(i)} \lambda^{i} & \sum_{i=0}^{7} k_{22}^{(i)} \lambda^{i} & \sum_{i=0}^{6} k_{23}^{(i)} \lambda^{i} & \sum_{i=0}^{7} k_{24}^{(i)} \lambda^{i} \\
0 & 0 & \sum_{i=0}^{7} k_{33}^{(i)} \lambda^{i} & \sum_{i=0}^{6} k_{34}^{(i)} \lambda^{i} \\
0 & 0 & \sum_{i=0}^{6} k_{43}^{(i)} \lambda^{i} & \sum_{i=0}^{7} k_{44}^{(i)} \lambda^{i}
\end{array}\right),
$$

where undetermined $k_{i j}^{(k)}$ are not functions of $\lambda$. The Equation (3.17) can be rewritten as,

$$
T_{t}+T U=K(\lambda) T .
$$

Substitute Equation (3.5) and Equation (3.18) into Equation (3.19). By comparing the coefficients of the same power of $\lambda$ on both sides of Equation (3.19) we can get Proposition 2. Because the results are very complex, we do not give them here.

In this section, we construct the Darboux transform of the high order nonlocal coupled AKNS system and establish the relationship between the new and old potentials. In the next section we will construct the exact solution of (2.6) using the results of this Section.

\section{Exact Solution of High Order Nonlocal Coupled AKNS System}

According to the classical Darboux transformation, firstly, four basic solutions of lax pairs (2.1) are given,

$$
\Delta=\left(\begin{array}{lll}
\phi\left(\lambda_{j}\right) & \psi\left(\lambda_{j}\right) & \Phi\left(\lambda_{j}\right)
\end{array} \quad \Psi\left(\lambda_{j}\right)\right)=\left(\begin{array}{llll}
\phi_{1} & \psi_{1} & \Phi_{1} & \Psi_{1} \\
\phi_{2} & \psi_{2} & \Phi_{2} & \Psi_{2} \\
\phi_{3} & \psi_{3} & \Phi_{3} & \Psi_{3} \\
\phi_{4} & \psi_{4} & \Phi_{4} & \Psi_{4}
\end{array}\right) .
$$

We know that by the Darboux transformation,

$$
\varphi^{[1]}=T^{[1]} \varphi=\left(\begin{array}{llll}
T_{11} & T_{12} & T_{13} & T_{14} \\
T_{21} & T_{22} & T_{23} & T_{24} \\
T_{31} & T_{23} & T_{33} & T_{34} \\
T_{41} & T_{42} & T_{43} & T_{44}
\end{array}\right)\left(\begin{array}{llll}
\phi_{1} & \psi_{1} & \Phi_{1} & \Psi_{1} \\
\phi_{2} & \psi_{2} & \Phi_{2} & \Psi_{2} \\
\phi_{3} & \psi_{3} & \Phi_{3} & \Psi_{3} \\
\phi_{4} & \psi_{4} & \Phi_{4} & \Psi_{4}
\end{array}\right),
$$

when $\lambda=\lambda_{j}$, the 4 columns of $\varphi^{[1]}$ are linear correlation. In other words, there are different constants $\gamma_{j}^{(1)}, \gamma_{j}^{(2)}, \gamma_{j}^{(3)}$ that satisfy the following relationship, 


$$
\left\{\begin{array}{l}
\sum_{j=1}^{4} T_{1 j} \phi_{j}+\gamma_{j}^{(1)} \sum_{j=1}^{4} T_{1 j} \psi_{j}+\gamma_{j}^{(2)} \sum_{j=1}^{4} T_{1 j} \Phi_{j}+\gamma_{j}^{(3)} \sum_{j=1}^{4} T_{1 j} \Psi_{j}=0 \\
\sum_{j=1}^{4} T_{2 j} \phi_{j}+\gamma_{j}^{(1)} \sum_{j=1}^{4} T_{2 j} \psi_{j}+\gamma_{j}^{(2)} \sum_{j=1}^{4} T_{2 j} \Phi_{j}+\gamma_{j}^{(3)} \sum_{j=1}^{4} T_{2 j} \Psi_{j}=0 \\
\sum_{j=1}^{4} T_{3 j} \phi_{j}+\gamma_{j}^{(1)} \sum_{j=1}^{4} T_{3 j} \psi_{j}+\gamma_{j}^{(2)} \sum_{j=1}^{4} T_{3 j} \Phi_{j}+\gamma_{j}^{(3)} \sum_{j=1}^{4} T_{3 j} \Psi_{j}=0 \\
\sum_{j=1}^{4} T_{4 j} \phi_{j}+\gamma_{j}^{(1)} \sum_{j=1}^{4} T_{4 j} \psi_{j}+\gamma_{j}^{(2)} \sum_{j=1}^{4} T_{4 j} \Phi_{j}+\gamma_{j}^{(3)} \sum_{j=1}^{4} T_{4 j} \Psi_{j}=0
\end{array}\right.
$$

Substitute (3.5) into (4.3), we get,

$$
\begin{aligned}
& b_{11}^{[1]}+\alpha_{j}^{(1)} b_{12}^{[1]}+\alpha_{j}^{(2)} b_{13}^{[1]}+\alpha_{j}^{(3)} b_{14}^{[1]}=-\lambda_{j}\left(1+\alpha_{j}^{(2)}\right) \\
& b_{21}^{[1]}+\alpha_{j}^{(1)} b_{22}^{[1]}+\alpha_{j}^{(2)} b_{23}^{[1]}+\alpha_{j}^{(3)} b_{24}^{[1]}=-\lambda_{j}\left(\alpha_{j}^{(1)}+\alpha_{j}^{(3)}\right) \\
& \alpha_{j}^{(2)} b_{11}^{[1]}+\alpha_{j}^{(3)} b_{12}^{[1]}=-\lambda_{j} \alpha_{j}^{(2)} \\
& \alpha_{j}^{(2)} b_{21}^{[1]}+\alpha_{j}^{(3)} b_{22}^{[1]}=-\lambda_{j} \alpha_{j}^{(3)}
\end{aligned}
$$

where

$$
\begin{aligned}
& \alpha_{j}^{(1)}=\frac{\gamma_{j}^{(1)} \psi_{2}+\gamma_{j}^{(2)} \Phi_{2}+\gamma_{j}^{(3)} \Psi_{2}+\phi_{2}}{\gamma_{j}^{(1)} \psi_{1}+\gamma_{j}^{(2)} \Phi_{1}+\gamma_{j}^{(3)} \Psi_{1}+\phi_{1}}, \quad \alpha_{j}^{(2)}=\frac{\gamma_{j}^{(1)} \psi_{3}+\gamma_{j}^{(2)} \Phi_{3}+\gamma_{j}^{(3)} \Psi_{3}+\phi_{3}}{\gamma_{j}^{(1)} \psi_{1}+\gamma_{j}^{(2)} \Phi_{1}+\gamma_{j}^{(3)} \Psi_{1}+\phi_{1}}, \\
& \alpha_{j}^{(3)}=\frac{\gamma_{j}^{(1)} \psi_{4}+\gamma_{j}^{(2)} \Phi_{4}+\gamma_{j}^{(3)} \Psi_{4}+\phi_{4}}{\gamma_{j}^{(1)} \psi_{1}+\gamma_{j}^{(2)} \Phi_{1}+\gamma_{j}^{(3)} \Psi_{1}+\phi_{1}}, \quad j=1,2 .
\end{aligned}
$$

Expression (4.4) can be written as a product of determinants,

$$
A X^{\mathrm{T}}=b^{\mathrm{T}},
$$

where

$$
A=\left(\begin{array}{cccccccc}
1 & \alpha_{1}^{(1)} & \alpha_{1}^{(2)} & \alpha_{1}^{(3)} & 0 & 0 & 0 & 0 \\
0 & 0 & 0 & 0 & 1 & \alpha_{1}^{(1)} & \alpha_{1}^{(2)} & \alpha_{1}^{(3)} \\
\alpha_{1}^{(2)} & \alpha_{1}^{(3)} & 0 & 0 & 0 & 0 & 0 & 0 \\
0 & 0 & 0 & 0 & \alpha_{1}^{(2)} & \alpha_{1}^{(3)} & 0 & 0 \\
1 & \alpha_{2}^{(1)} & \alpha_{2}^{(2)} & \alpha_{3}^{(3)} & 0 & 0 & 0 & 0 \\
0 & 0 & 0 & 0 & 1 & \alpha_{2}^{(1)} & \alpha_{2}^{(2)} & \alpha_{2}^{(3)} \\
\alpha_{2}^{(2)} & \alpha_{2}^{(3)} & 0 & 0 & 0 & 0 & 0 & 0 \\
0 & 0 & 0 & 0 & \alpha_{2}^{(2)} & \alpha_{3}^{(3)} & 0 & 0
\end{array}\right),
$$

and

$$
\begin{aligned}
& X=\left(\begin{array}{llllllll}
b_{11}^{[1]} & b_{12}^{[1]} & b_{13}^{[1]} & b_{14}^{[1]} & b_{21}^{[1]} & b_{22}^{[1]} & b_{23}^{[1]} & b_{24}^{[1]}
\end{array}\right), \\
& b=\left(\begin{array}{llllllll}
-\lambda_{1}\left(1+\alpha_{1}^{(2)}\right) & -\lambda_{1}\left(\alpha_{1}^{(1)}+\alpha_{1}^{(3)}\right) & -\lambda_{1} \alpha_{1}^{(2)} & -\lambda_{1} \alpha_{1}^{(3)} & -\lambda_{2}\left(1+\alpha_{2}^{(2)}\right) & -\lambda_{2}\left(\alpha_{2}^{(1)}+\alpha_{2}^{(3)}\right) & -\lambda_{2} \alpha_{2}^{(2)} & -\lambda_{2} \alpha_{2}^{(3)}
\end{array}\right) .
\end{aligned}
$$

To get $b_{1 i}^{[1]}, b_{2 i}^{[1]}, i=1,2,3,4$, we need to solve the system (4.6), but, we need to give the basic solutions for Lax pair firstly,

$$
\phi\left(\lambda_{j}\right)=\left(c_{j}^{(1)} \lambda_{j}\left(\beta \lambda_{j}^{2} t-x\right) \mathrm{e}^{\alpha \lambda_{j}^{3} t-\lambda_{j} x}, 0, c_{j}^{(1)} \mathrm{e}^{\alpha \lambda_{j}^{3} t-\lambda_{j} x}, 0\right)^{\mathrm{T}},
$$




$$
\begin{aligned}
& \psi\left(\lambda_{j}\right)=\left(0,-c_{j}^{(2)} \lambda_{j}\left(\beta \lambda_{j}^{2} t-x\right) \mathrm{e}^{\lambda_{j} x-\alpha \lambda_{j}^{3} t}, 0, c_{j}^{(2)} \mathrm{e}^{\lambda_{j} x-\alpha \lambda_{j}^{3} t}\right)^{\mathrm{T}}, \\
& \Phi\left(\lambda_{j}\right)=\left(c_{j}^{(3)} \mathrm{e}^{\alpha \lambda_{j}^{3} t-\lambda_{j} x}, 0,0,0\right)^{\mathrm{T}}, \\
& \Psi\left(\lambda_{j}\right)=\left(0, c_{j}^{(4)} \mathrm{e}^{\lambda_{j} x-\alpha \lambda_{j}^{3} t}, 0,0,0\right)^{\mathrm{T}},
\end{aligned}
$$

with $c_{j}^{(k)}(j=1,2 ; k=1,2,3,4)$ are arbitrary constants.

By solving system of linear Equation (4.6) and using Equations (4.5), (4.7), we can get the expression of $b_{12}^{[1]}(x, t), b_{21}^{[1]}(x, t)$ and $b_{14}^{[1]}(x, t), b_{23}^{[1]}(x, t)$ since only these four variables are included in the Darboux transformation.

$$
\begin{aligned}
& b_{12}^{[1]}(x, t)=\frac{c_{1}^{(1)} c_{1}^{(2)}\left(\lambda_{2}-\lambda_{1}\right) \mathrm{e}^{\left(\lambda_{1}+\lambda_{2}\right)\left(\alpha \lambda_{1}^{2} t+\alpha \lambda_{2}^{2} t-\alpha \lambda_{1} \lambda_{2} t-x\right)}}{c_{1}^{(1)} c_{2}^{(2)} \gamma_{2} \mathrm{e}^{\left(\lambda_{2}^{(1)}-\lambda_{1}\right)\left(\alpha \lambda_{1}^{2} t+\alpha \lambda_{2}^{2} t+\alpha \lambda_{1} \lambda_{2} t-x\right)}-c_{1}^{(2)} c_{2}^{(1)} \gamma_{1}^{(1)} \mathrm{e}^{\left(\lambda_{1}-\lambda_{2}\right)\left(\alpha \lambda_{1}^{2} t+\alpha \lambda_{2}^{2} t+\alpha \lambda_{1} \lambda_{2} t-x\right)}}, \\
& b_{21}^{[1]}(x, t)=-\frac{c_{2}^{(1)} c_{2}^{(2)} \gamma_{1}^{(1)} \gamma_{2}^{(1)}\left(\lambda_{2}-\lambda_{1}\right) \mathrm{e}^{-\left(\lambda_{1}+\lambda_{2}\right)\left(\alpha \lambda_{1}^{2} t+\alpha \lambda_{2}^{2} t-\alpha \lambda_{1} \lambda_{2} t-x\right)}}{c_{1}^{(1)} c_{2}^{(2)} \gamma_{2}^{(1)} \mathrm{e}^{\left(\lambda_{2}-\lambda_{1}\right)\left(\alpha \lambda_{1}^{2} t+\alpha \lambda_{2}^{2} t+\alpha \lambda_{1} \lambda_{2} t-x\right)}-c_{1}^{(2)} c_{2}^{(1)} \gamma_{1}^{(1)} \mathrm{e}^{\left(\lambda_{1}-\lambda_{2}\right)\left(\alpha \lambda_{1}^{2} t+\alpha \lambda_{2}^{2} t+\alpha \lambda_{1} \lambda_{2} t-x\right)},} \\
& b_{14}^{[1]}(x, t)=\frac{\Delta_{1} \mathrm{e}^{2 \lambda_{2}\left(\alpha \lambda_{2}^{2} t-x\right)}+\Delta_{2} \mathrm{e}^{2 \lambda_{1}\left(\alpha \lambda_{1}^{2} t-x\right)}}{c_{1}^{(1)} c_{2}^{(2)}\left(\gamma_{1}^{(2)}\right)^{2} \mathrm{e}^{2\left(\lambda_{2}-\lambda_{1}\right)\left(\alpha \lambda_{1}^{2} t+\alpha \lambda_{2}^{2} t+\alpha \lambda_{1} \lambda_{2} t-x\right)}+c_{1}^{(2)} c_{2}^{(1)}\left(\gamma_{1}^{(1)}\right)^{2} \mathrm{e}^{-2\left(\lambda_{1}-\lambda_{2}\right)\left(\alpha \lambda_{1}^{2} t+\alpha \lambda_{2}^{2} t+\alpha \lambda_{1} \lambda_{2} t-x\right)}-2 \gamma_{1}^{(2)} \gamma_{1}^{(1)} c_{1}^{(1)} c_{2}^{(2)} c_{1}^{(2)} c_{2}^{(1)}}, \\
& b_{23}^{[1]}(x, t)=-\frac{\Delta_{3} \mathrm{e}^{-2 \lambda_{2}\left(\alpha \lambda_{2}^{2} t-x\right)}+\Delta_{4} \mathrm{e}^{-2 \lambda_{1}\left(\alpha \lambda_{1}^{2} t-x\right)}}{\left(c_{1}^{(1)} c_{2}^{(2)} \gamma_{1}^{(2)} \mathrm{e}^{\left(\lambda_{2}-\lambda_{1}\right)\left(\alpha \lambda_{1}^{2} t+\alpha \lambda_{2}^{2} t+\alpha \lambda_{1} \lambda_{2} t-x\right)}-c_{1}^{(2)} c_{2}^{(1)} \gamma_{1}^{(1)} \mathrm{e}^{-\left(\lambda_{1}-\lambda_{2}\right)\left(\alpha \lambda_{1}^{2} t+\alpha \lambda_{2}^{2} t+\alpha \lambda_{1} \lambda_{2} t-x\right)}\right)^{2}},
\end{aligned}
$$

where

$$
\begin{aligned}
\Delta_{1}= & \left(c_{1}^{(1)}\right)^{2}\left(\lambda_{2}-\lambda_{1}\right)\left[2 \gamma_{1}^{(2)} c_{1}^{(2)} c_{2}^{(2)}\left(\beta \lambda_{1}^{3} t-\lambda_{1} x\right)\right. \\
& \left.+\gamma_{1}^{(2)} c_{2}^{(2)}\left(\gamma_{2}^{(2)} c_{3}^{(2)}+c_{1}^{(2)}\right)-\gamma_{3}^{(2)} c_{1}^{(2)} c_{4}^{(2)}\right], \\
\Delta_{2}= & -\left(c_{1}^{(2)}\right)^{2}\left(\lambda_{2}-\lambda_{1}\right)\left[2 \gamma_{1}^{(1)} c_{1}^{(1)} c_{2}^{(1)}\left(\beta \lambda_{2}^{3} t-\lambda_{2} x\right)\right. \\
& \left.+\gamma_{1}^{(1)} c_{2}^{(1)}\left(\gamma_{2}^{(1)} c_{3}^{(1)}+c_{1}^{(1)}\right)-\gamma_{3}^{(1)} c_{1}^{(1)} c_{4}^{(1)}\right], \\
\Delta_{3}= & \left(c_{2}^{(1)} \gamma_{1}^{(1)}\right)^{2}\left(\lambda_{2}-\lambda_{1}\right)\left[2 \gamma_{1}^{(2)} c_{1}^{(2)} c_{2}^{(2)}\left(\beta \lambda_{1}^{3} t-\lambda_{1} x\right)\right. \\
& \left.+\gamma_{1}^{(2)} c_{2}^{(2)}\left(\gamma_{2}^{(2)} c_{3}^{(2)}-c_{1}^{(2)}\right)-\gamma_{3}^{(2)} c_{1}^{(2)} c_{4}^{(2)}\right], \\
\Delta_{4}= & -\left(c_{1}^{(2)} \gamma_{1}^{(2)}\right)^{2}\left(\lambda_{2}-\lambda_{1}\right)\left[2 \gamma_{1}^{(1)} c_{1}^{(1)} c_{2}^{(1)}\left(\beta \lambda_{2}^{3} t-\lambda_{2} x\right)\right. \\
& \left.+\gamma_{1}^{(1)} c_{2}^{(1)}\left(\gamma_{2}^{(1)} c_{3}^{(1)}-c_{1}^{(1)}\right)-\gamma_{3}^{(1)} c_{1}^{(1)} c_{4}^{(1)}\right] .
\end{aligned}
$$

Using the symmetry reduction condition (3.8), we can get

$$
\gamma_{1}^{(2)}=i \frac{c_{1}^{(2)}}{c_{2}^{(2)}}, \gamma_{3}^{(2)}=i \frac{c_{3}^{(2)} \gamma_{2}^{(2)}}{c_{4}^{(2)}}, \gamma_{1}^{(1)}=-i \frac{c_{1}^{(1)}}{c_{2}^{(1)}}, \gamma_{3}^{(1)}=-i \frac{c_{3}^{(1)} \gamma_{2}^{(1)}}{c_{4}^{(1)}},
$$

where $i^{2}=-1$. In this case, we obtain the new solution,

$$
\begin{aligned}
& p^{[1]}=2 b_{12}^{[1]}(x, t), \\
& r^{[1]}=2 b_{14}^{[1]}(x, t),
\end{aligned}
$$


with $\lambda_{1}, \lambda_{2}, c_{1}^{(1)}, c_{1}^{(2)}, c_{2}^{(1)}, c_{3}^{(1)}, c_{2}^{(2)}, c_{3}^{(2)}, c_{4}^{(1)}, c_{4}^{(2)}$ are arbitrary constants, $\gamma_{1}^{(2)}, \gamma_{3}^{(2)}, \gamma_{1}^{(1)}, \gamma_{3}^{(1)}$ conditions satisfied (4.9).

Through verification, we can know that solutions (4.10) are exact solutions of high order nonlocal coupled AKNS system (2.6). It can be seen from the expression (4.8) that the solutions of the system are in the form of exponential function, and the soliton solutions of the nonlocal system can be constructed by selecting appropriate parameters. In order to study the characteristics of the solutions, the graphs of module $p$ and $r$ are illustrated in Figure 1 and Figure 2 by selecting the appropriate parameters.

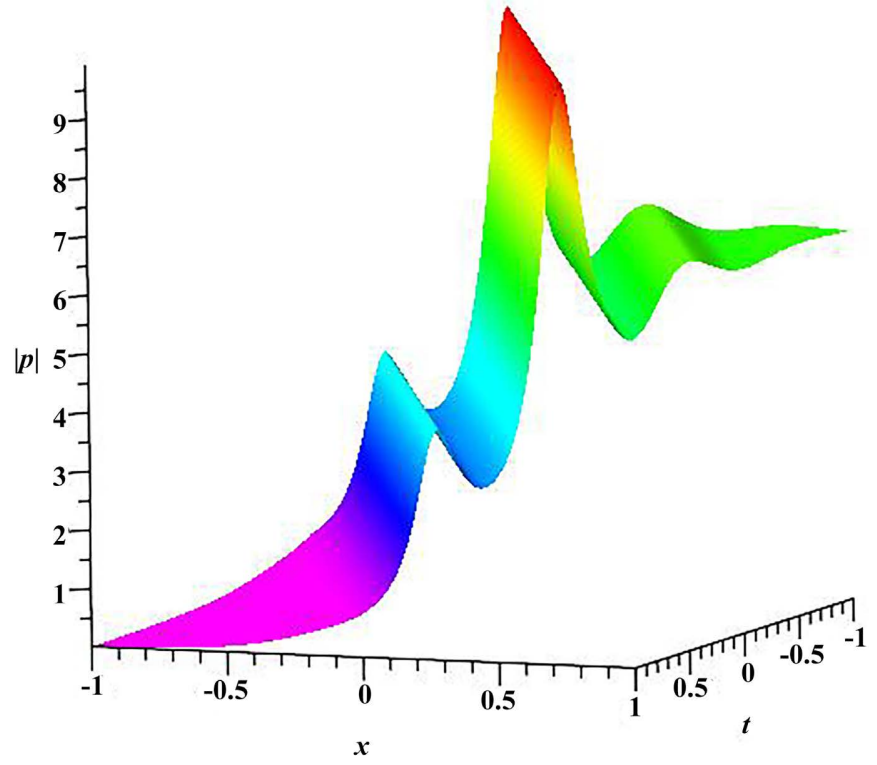

(a)

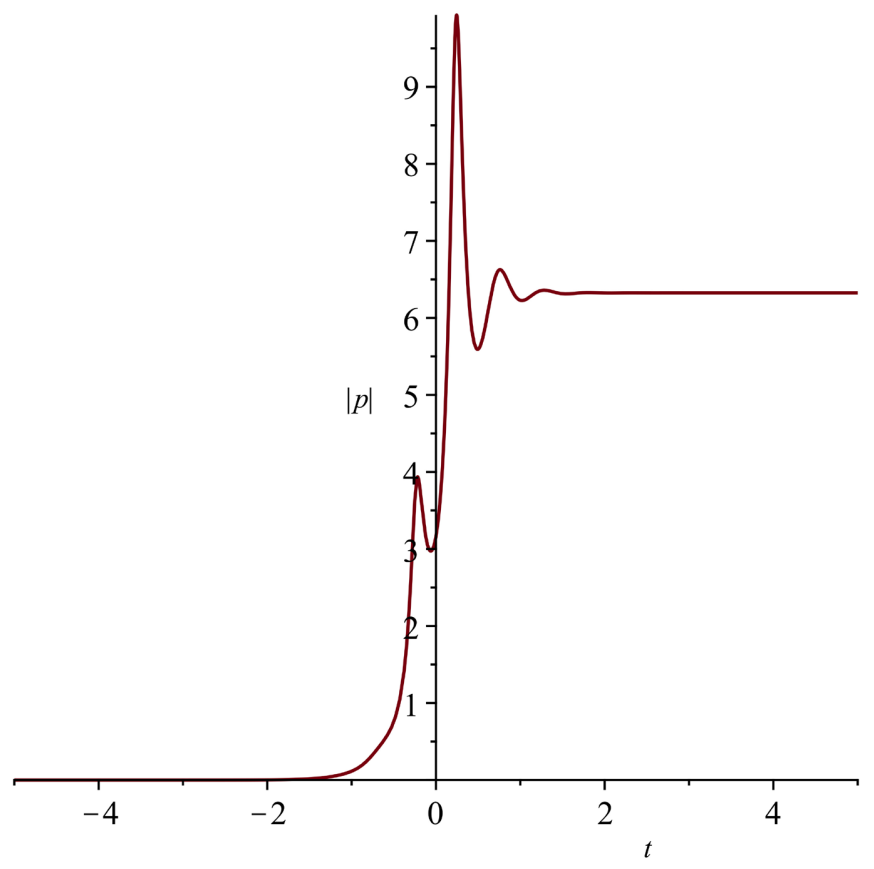

(b) 


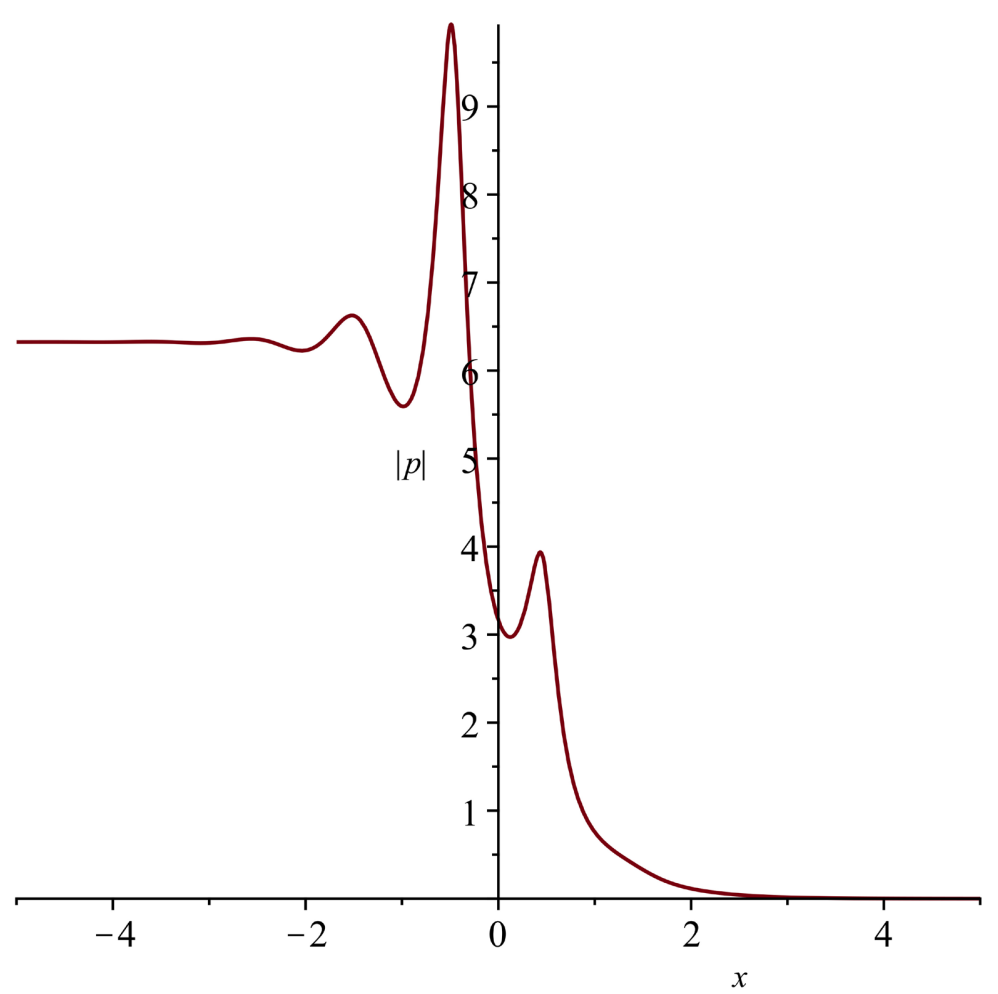

(c)

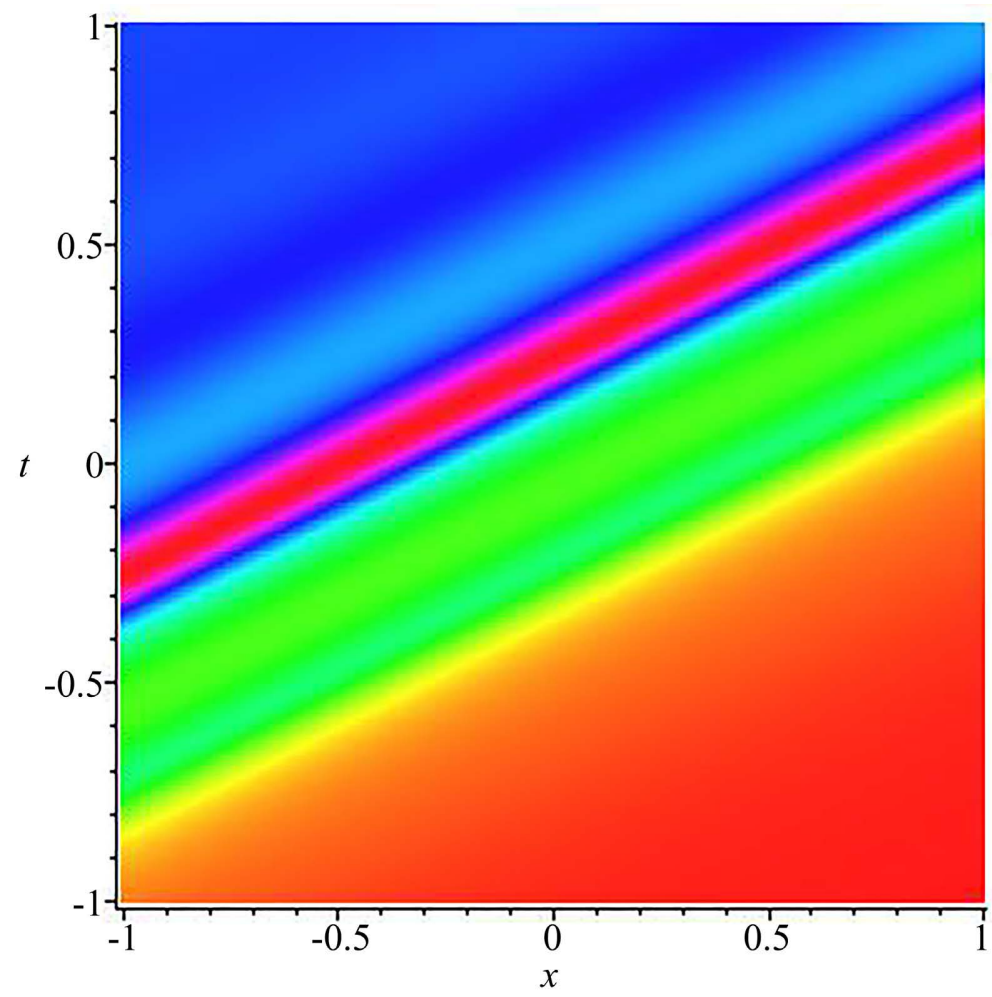

(d)

Figure 1. (a) The modulus of $P^{[1]}$ in expression (4.10) with the parameters $\lambda_{1}=1-i$, $\lambda_{1}=2 i, \alpha_{2}=-1$. (b) 2D graph of expression (4.10) with $x=0$. (c) 2D graph of expression (4.10) with $t=0$. (d) Density graph of expression (4.10). 


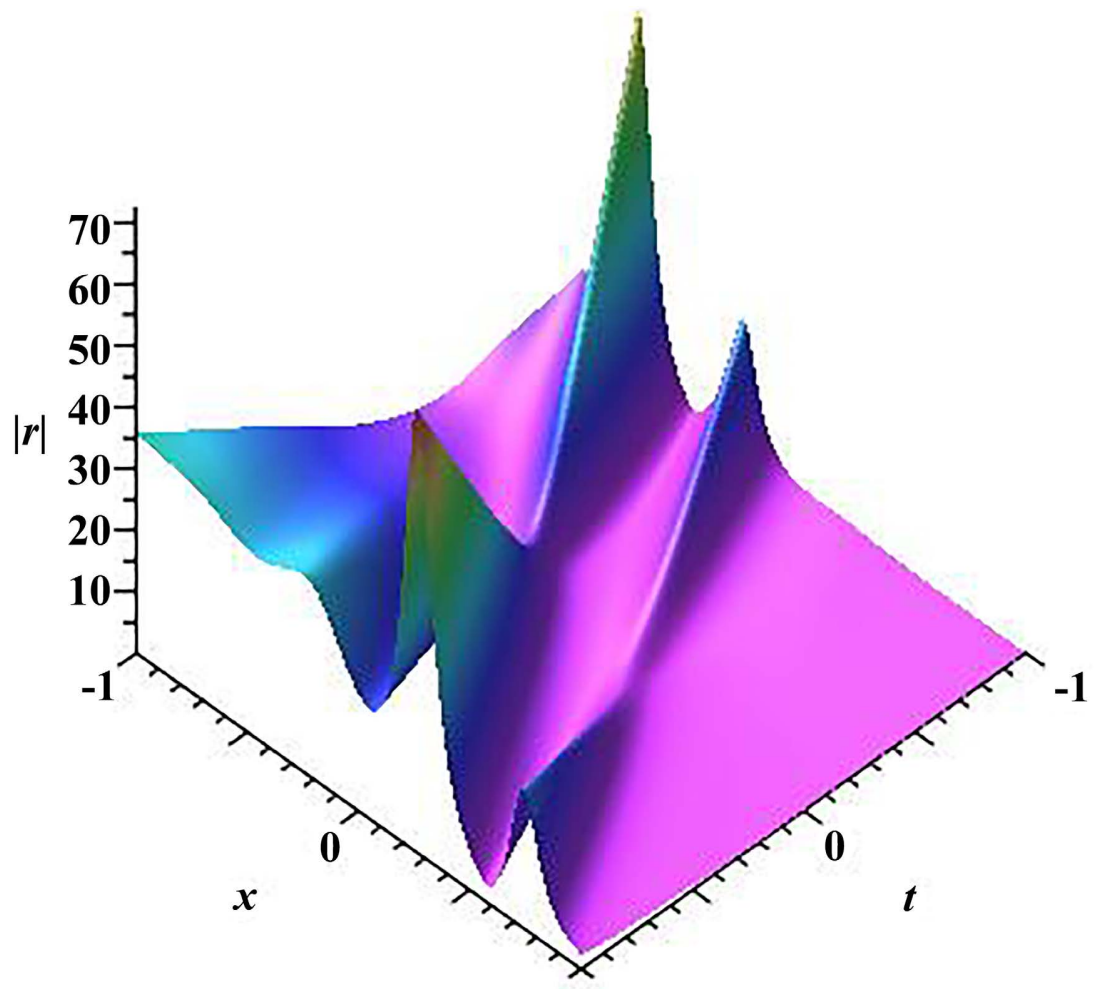

(a)

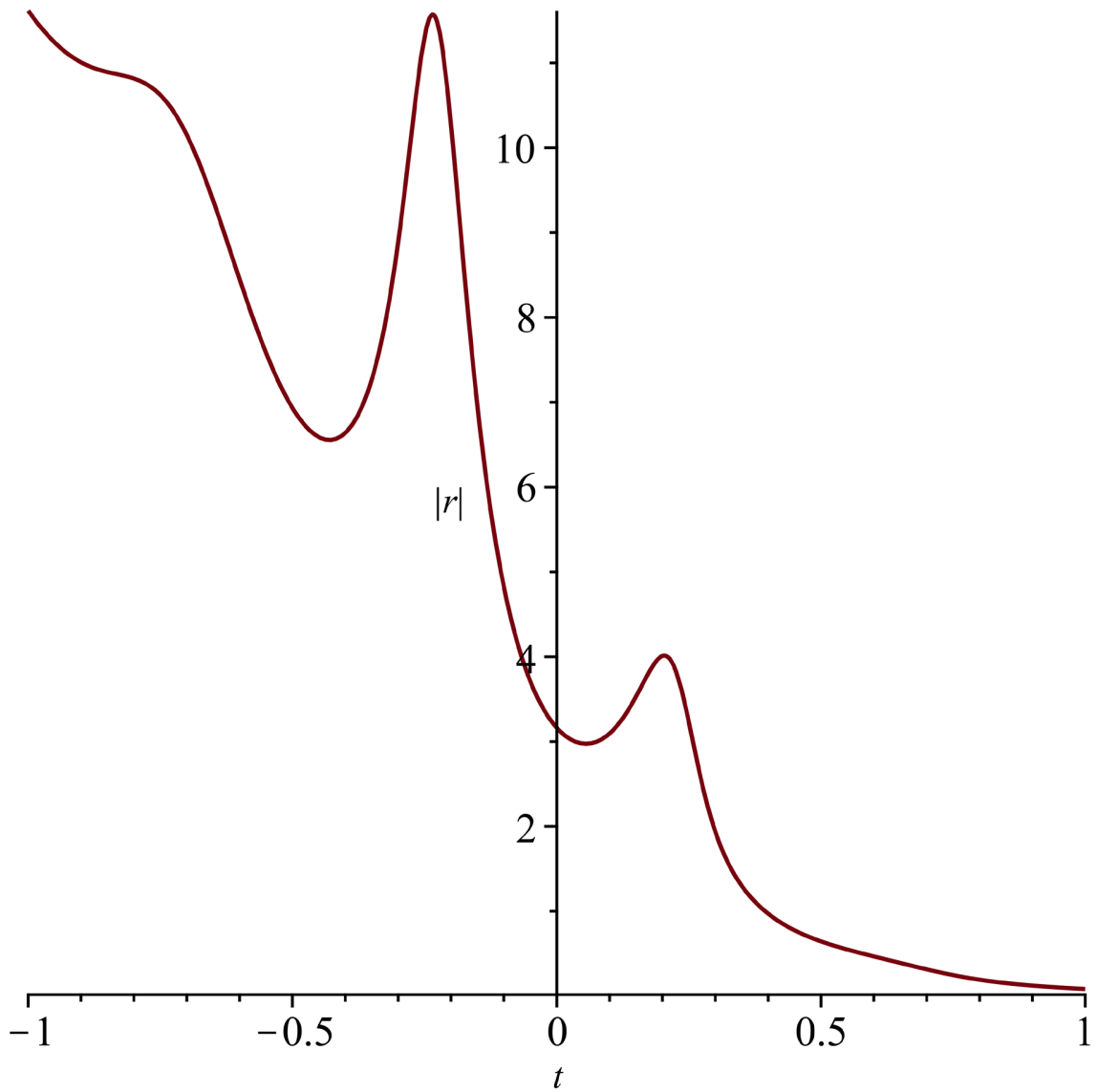

(b) 


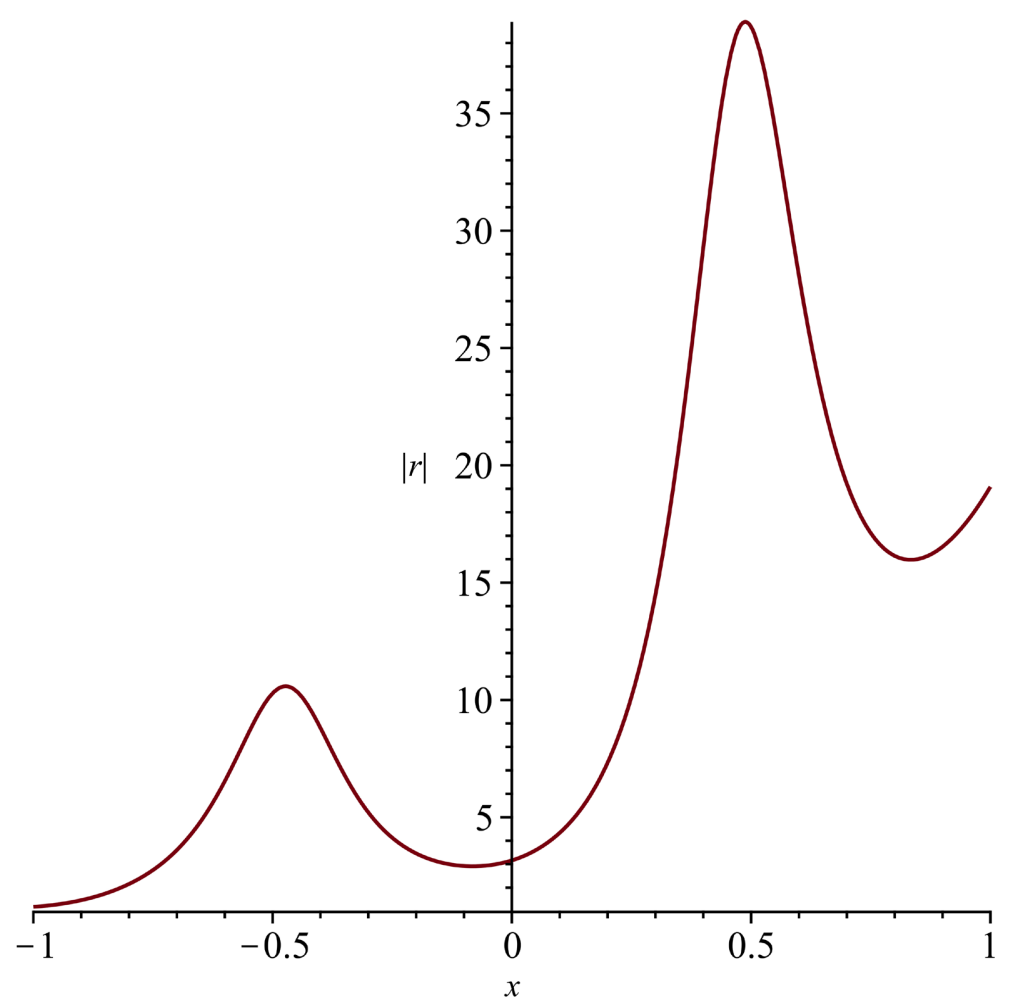

(c)

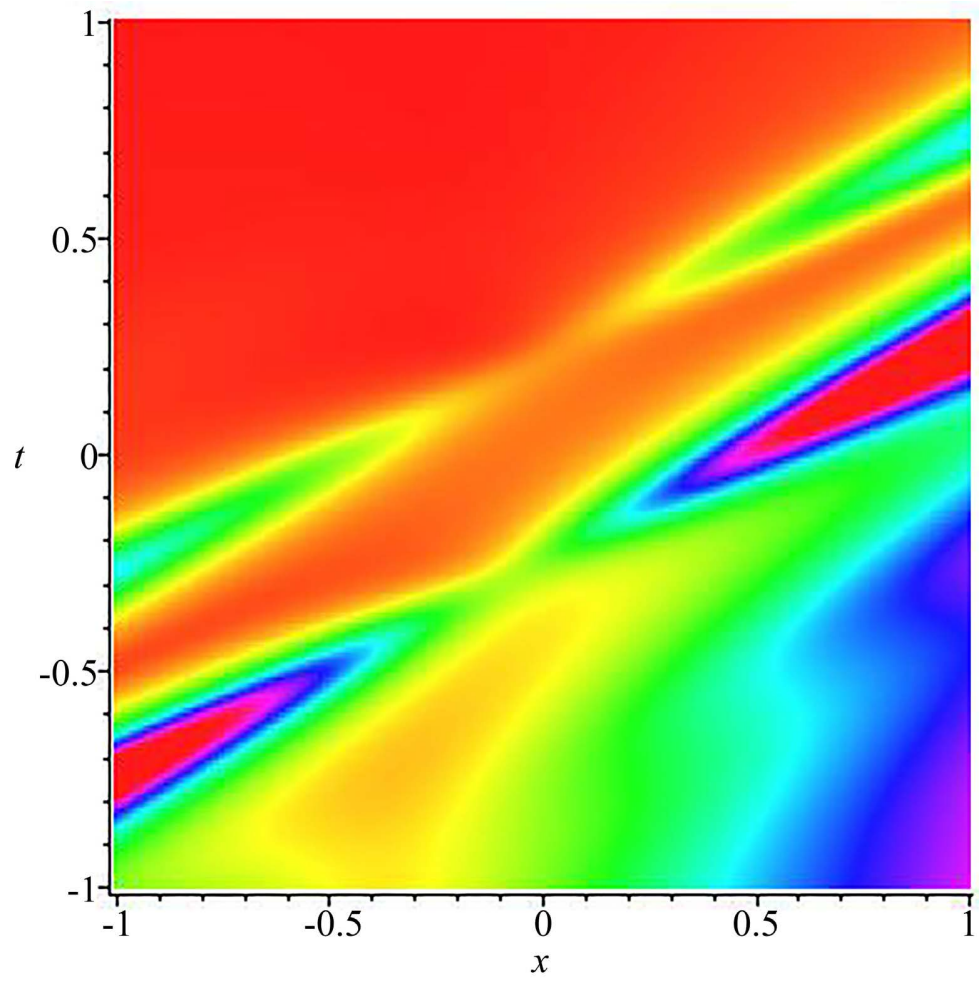

(d)

Figure 2. (a) The modulus of $r^{[1]}$ in expression (4.10) with the parameters $\lambda_{1}=1-i$, $\lambda_{1}=2 i, \quad \alpha_{2}=-1, \beta=-0.1$. (b) 2D graph of expression (4.10) with $x=0$. (c) 2D graph of expression (4.10) with $t=0$. (d) Density graph of expression (4.10). 
We know that if this solution is taken further as a new solution, we can perform the Darboux transformation again to produce another new solution. This process can be carried out continuously and a series of soliton solutions for high order nonlocal coupled AKNS system (2.6) can be theoretically obtained.

\section{Summary and Discussion}

In this paper, considering the practical importance of nonlocal equations, a high-order nonlocal coupled AKNS system is constructed and studied by using the symmetric reduction method for the first time. With the help of lax pairs, the gauge transformation of the system is constructed, and the Darboux transformation of the system is obtained. In this paper, a new exact solution of the system is constructed by using the trivial seed solution and Darboux transformation, and the soliton solution of the system is obtained by selecting appropriate parameters. In order to study the properties of soliton solution, the corresponding images are given.

It is very meaningful to study the coupled nonlocal integrable model. However, much remains to be done. For example, the construction of Lie symmetry, symmetry reduction and similar solutions of nonlocal equations should be studied. In addition, the rogue wave solution of nonlocal equations is one of the research hotspots in recent years. Therefore, it is very meaningful to construct the rogue wave solution of nonlocal equations with variable coefficients by using the Darboux transform method. The above topics will be discussed in a series of future research works.

\section{Acknowledgements}

This work is supported by the National Natural Science Foundation of China (Nos. 11505090, 1171041), the Natural Science Foundation of Shaanxi Province under Grant No. 2018JQ-1045, and the Science and Technology Innovation Foundation of Xian under Grant No. 2017CGWL06. We also acknowledge Research Award Foundation for Outstanding Young Scientists of Shandong Province (No. BS2015SF009) and the doctoral foundation of Liaocheng University under Grant No. 318051413.

\section{Conflicts of Interest}

The authors declare no conflicts of interest regarding the publication of this paper.

\section{References}

[1] Zakharov, V.E. (1980) The Inverse Scattering Method. In: Bullough, R.K. and Caudrey P.J., Eds., Solitons: Topics in Current Physics. Springer, Berlin, 243-285. https://doi.org/10.1007/978-3-642-81448-8_7

[2] Ablowitz, M.J., Ablowitz, M.A., Clarkson, P.A., et al. (1991) Solitons, Nonlinear Evolution Equations and Inverse Scattering. Cambridge University Press, Cambridge. https://doi.org/10.1017/CBO9780511623998 
[3] Zhang, Z.C. and Fan, E.G. (2021) Inverse Scattering Transform and Multiple High-Order Pole Solutions for the Gerdjikov-Ivanov Equation under the Zero/Nonzero Background. Zeitschrift Für Angewandte Mathematik und Physik, 72, Article No. 153. https://doi.org/10.1007/s00033-021-01583-x

[4] Guo B.L., Ling L.M. and Liu Q.P. (2013) High-Order Solutions and Generalized Darboux Transformations of Derivative Nonlinear Schrödinger Equations. Studies in Applied Mathematics, 130, 317-344.

https://doi.org/10.1111/j.1467-9590.2012.00568.x

[5] Duan, C.N. and Yu, F.J. (2018) N-Fold Darboux Transformation for the Nonlocal Nonlinear Schrödinger (NNLS) Equation with the Self-Induced PT-Symmetric Potential. Journal of Applied Mathematics and Physics, 6, 888-900.

https://doi.org/10.4236/jamp.2018.64076

[6] Chen, J.C., Ma, Z.Y. and Hu, Y.H. (2018) Nonlocal Symmetry, Darboux Transformation and Soliton-Cnoidal Wave Interaction Solution for the Shallow Water Wave Equation. Journal of Mathematical Analysis and Applications, 460, 987-1003. https://doi.org/10.1016/j.jmaa.2017.12.028

[7] Chen, J. (2015) Generalized Darboux Transformation and Rational Solutions for the Nonlocal Nonlinear Schrödinger Equation with the Self-Induced Parity-Time Symmetric Potential. Journal of Applied Mathematics and Physics, 3, 2327-4352. https://doi.org/10.4236/jamp.2015.35065

[8] Schnizer, W.A. and Leeb, H. (1993) Exactly Solvable Models for the Schrodinger Equation from Generalized Darboux Transformations. Journal of Physics A: Mathematical and General, 26, Article ID: 5145. https://doi.org/10.1088/0305-4470/26/19/041

[9] Hirota, R. (2004) The Direct Method in Soliton Theory. Cambridge University Press, Cambridge. https://doi.org/10.1017/CBO9780511543043

[10] Milnor, J.W. and Husemoller, D. (1973) Symmetric Bilinear Forms. Springer, Berlin. https://doi.org/10.1007/978-3-642-88330-9

[11] Hietarinta, J. (2005) Hirota's Bilinear Method and Soliton Solutions. Physics AUC, 15, 31-37.

[12] Zhao, X., Tian B., et al. (2021) Bilinear Bäcklund Transformation, Lax Pair and Interactions of Nonlinear Waves for a Generalized $(2+1)$-Dimensional Nonlinear Wave Equation in Nonlinear Optics/Fluid Mechanics/Plasma Physics. Nonlinear Dynamics, 103, 1785-1794. https://doi.org/10.1007/s11071-020-06154-9

[13] Ablowitz, M.J. and Musslimani, Z.H. (2013) Integrable Nonlocal Nonlinear Schrödinger Equation. Physical Review Letters, 110, Article ID: 064105. https://doi.org/10.1103/PhysRevLett.110.064105

[14] Nelson, O., Yu, Z., Dorian, B. and Wang, Y. (2018) A New Method for the Exact Solution of Duffing Equation. Journal of Applied Mathematics and Physics, 6, 2718-2726. https://doi.org/10.4236/jamp.2018.612225

[15] Siddiqui, A., Walait, A., Allison, T. and Haroon, T. (2018) Exact Solution for Pressure Driven Flow of Two Immiscible Phan-Thien-Tanner Fluids in a Pipe. Open Journal of Fluid Dynamics, 8, 378-391. https://doi.org/10.4236/ojfd.2018.84024

[16] Rao, J., He, J., et al. (2020) PT-Symmetric Nonlocal Davey-Stewartson I Equation: General Lump-Soliton Solutions on a Background of Periodic Line Waves. Applied Mathematics Letters, 104, Article ID: 106246. https://doi.org/10.1016/j.aml.2020.106246

[17] Yan, Z.Y. (2016) Nonlocal General Vector Nonlinear Schrödinger Equations: Integrability, PTSymmetribility, and Solutions. Applied Mathematics Letters, 62, 101-109. 
https://doi.org/10.1016/j.aml.2016.07.010

[18] Chen, J., Yan, Q. and Zhang, H. (2020) Multiple Bright Soliton Solutions of a Reverse-Space Nonlocal Nonlinear Schrödinger Equation. Applied Mathematics Letters, 106, Article ID: 106375. https://doi.org/10.1016/j.aml.2020.106375

[19] Ji, J.L. and Zhu, Z.N. (2017) Soliton Solutions of an Integrable Nonlocal Modified Korteweg-de Vries Equation through Inverse Scattering Transform. Journal of Mathematical Analysis and Applications, 453, 973-984.

https://doi.org/10.1016/j.jmaa.2017.04.042

[20] Yang, Y.Q., Takashi, S. and Cheng, X.P. (2020) Darboux Transformations and Exact Solutions for the Integrable Nonlocal Lakshmanan-Porsezian-Daniel Equation. Applied Mathematics Letters, 99, Article ID: 105998.

https://doi.org/10.1016/j.aml.2019.105998

[21] Zhang, X.E., Chen, Y. and Zhang, Y. (2017) Breather, Lump and XSoliton Solutions to Nonlocal KP Equation. Computers \& Mathematics with Applications, 74, 2341-2347. https://doi.org/10.1016/j.camwa.2017.07.004

[22] Zhou, Z.X. (2018) Darboux Transformations and Global Explicit Solutions for Nonlocal Davey-Stewartson I Equation. Studies in Applied Mathematics, 141, 186-204. https://doi.org/10.1111/sapm.12219

[23] Huang, X. and Ling, L.M. (2016) Soliton Solutions for the Nonlocal Nonlinear Schrödinger Equation. The European Physical Journal Plus, 131, Article No. 148. https://doi.org/10.1140/epjp/i2016-16148-9

[24] Zhang, Y.X., et al. (2008) Lax Pair and Darboux Transformation for a Variable-Coefficient Fifth-Order Korteweg-de Vries Equation with Symbolic Computation. Communications in Theoretical Physics, 49, Article ID: 833. https://doi.org/10.1088/0253-6102/49/4/06

[25] Zhou, Z.X. (2016) Darboux Transformations and Global Solutions for a Nonlocal Derivative Nonlinear Schrodinger Equation. Communications in Nonlinear Science Numerical Simulation, 62, 480-488. https://doi.org/10.1016/j.cnsns.2018.01.008

[26] Ma, W.X. and Zhang, Y.J. (2018) Darboux Transformations of Integrable Couplings and Applications. Reviews in Mathematical Physics, 30, Article ID: 1850003. https://doi.org/10.1142/S0129055X18500034 